\title{
EL ROL DEL PSICÓLOGO EN EL EQUIPO INTERDISCIPLINARIO DE CIRUGÍA BARIÁTRICA
}

\section{THE PSYCHOLOGIST'S ROLE IN A BARIATRIC INTERDISCIPLINARY TEAM}

\author{
Mariana Alejandra Sierra Murguía* \\ *Maestra en Psicología con Residencia en Medicina Conductual. Investigador A en Ciencias Médicas en la Clínica \\ de Obesidad del Hospital General Dr. Manuel Gea González. \\ E-Mail: mariana.sierra.m@gmail.com \\ Calzada de Tlalpan 4800, Sección XVI, CP 14080, México D.F., México.
}

\begin{abstract}
RESUMEN
La obesidad ha sido declarada por la OMS como una epidemia y es factor de riesgo para diversas enfermedades tanto físicas como psicológicas, por lo que supone una creciente carga económica para los sistemas de salud. Actualmente el tratamiento que mayor efectividad ha mostrado para tratar la obesidad severa es la cirugía bariátrica; sin embargo, a pesar de los cambios anatómicos realizados por la cirugía, no todos los pacientes se ven beneficiados por la misma. Se ha reportado que el $20 \%$ de estas cirugías fracasan y generalmente este fracaso es atribuido a causas psicológicas. El número de cirugías bariátricas realizadas en el mundo está aumentando exponencialmente y dentro de las guías norteamericanas se sugiere el tratamiento interdisciplinario, incluyendo en este el tratamiento psicológico. El trabajo que se informa busca describir el rol del psicólogo en un equipo interdisciplinario de cirugía bariátrica.
\end{abstract}

Palabras clave: Cirugía bariátrica; Psicología Bariátrica; Obesidad; Interdisciplina; Modificación de hábitos.

\section{ABSTRACT}

Obesity has been declared by the WHO as an epidemic. It has been described as a multicausal disease and that it's etiology is influenced by biological, psychological, environmental and other factors. It is a risk factor for many diseases not only physical (diabetes, hypertension, hypercholesterolemia, an so on) but also psychological (depression, anxiety, eating behavior disorders, etc.); reason for which it's supposed to be a raising charge for worldwide health systems. Among the approved treatments for overweight and obesity are included: nutritional (caloric restriction), increase physical activity and behavioral therapy. When talking about patients with severe obesity $(B M I \geq 40)$, the most effective treatment that has been proven is bariatric surgery, indicated for patients with a BMI $\geq 40$ or $\geq 35$ in case of presenting co morbidities. It has been described that surgical treatment for obesity promotes mayor weight loss, generates significant improvement of the co morbidities associated with the obese condition and helps to prolong life expectancy and to increase quality of life. Despite all of the advantages that this treatment has proven to offer, not all of the patients benefit from it, even with the anatomical changes made by the surgery, some of the patients don't get to loose a significant amount of weight; differences in the variations of weight after the surgery at short and long term have been reported. It has been reported that approximately $20 \%$ of bariatric surgeries fail due to poor weight loss and that this failure is generally attributed to psychological or behavioral causes. 
Nowadays the American Society for Metabolic and Bariatric Surgery (ASMBS) guidelines suggest that surgical treatment for obesity must be performed in a interdisciplinary approach that includes surgeon, nutritionist, internist, physical rehabilitator and a psychologist. According to the same guidelines, the psychologist's role in this team consists on initially evaluating surgery candidates in order to determine if there is existing psychopathology and their ability to implement the lifestyle changes needed before and after the procedure, to give psico educational interventions in order to prepare patients for these changes and to make an informed decision about this treatment. This preparation helps patients having realistic expectations about the surgery results, helps to decrease the uncertainty that generates anxiety. For this reason, psychologist's work should be focused on motivation and adherence on lifestyle changes by psycho educational and cognitive behavioral specific interventions. In conclusion, the goal of the psychologist in the bariatric surgery team is to identify possible contraindications for the surgery and to identify possible post-surgical challenges as well as to give behavioral specific strategies in order to cope with those challenges.

The prevalence of obesity is rising across the world and, in order to treat it, the increase in the number of bariatric surgeries performed. As ASMBS guidelines suggest a interdisciplinary approach where the psychologist plays a fundamental role in order to get a better prognosis after the surgery, it is important to increase the number of psychologists with the necessary skills to work with these patients, and with knowledge about obesity and bariatric surgery. The present research aims to describe the psychologist's role in a bariatric surgery interdisciplinary clinic and some of the strategies described for psychological obesity treatment suggested to be used before and after the surgery, in order to promote a lifestyle change.

Key words: Bariatric surgery; Bariatric Psychology; Obesity; Interdiscipline; Habit modification.

\section{INTRODUCCIÓN}

Desde hace más de una década, la Organización Mundial de la Salud (OMS, 2000) emitió un reporte en el que declaró una epidemia global de obesidad. Este reporte se basó en informes provenientes de diferentes grupos poblacionales en los que se observó una tendencia al aumento de las tasas de obesidad iniciado hace aproximadamente 60 años atrás.

La OMS (2006) define la obesidad y el sobrepeso como una acumulación anormal o excesiva de grasa que puede ser perjudicial para la salud. Es originada por diversas causas tales como biológicas, ambientales, conductuales y conlleva numerosas complicaciones. Se identifica con el índice de masa corporal (IMC), definido como la relación entre el peso y la talla $\left(\mathrm{kg} / \mathrm{m}^{2}\right)$, en donde se considera sobrepeso a un IMC igual o superior a 25 y obesidad, un IMC igual o superior a 30 .

Debido a que la obesidad representa un factor de riesgo para diversas enfermedades tales como diabetes, hipertensión, dislipidemias, entre otras, constituye un problema relevante en materia de salud pública a nivel mundial, que supone una creciente carga económica sobre los recursos nacionales; por lo tanto, es una tarea primordial de los organismos encargados de salud pública diseñar e instrumentar estrategias de prevención y tratamiento.

De acuerdo con informes de la OMS, en el año 2005 más de 1.600 millones de personas mayores de 15 años presentaban sobrepeso y al menos 400 millones, obesidad. Además, dicha organización calcula que para el 2015 habrá aproximadamente 2.300 millones de adultos con sobrepeso y más de 700 millones con obesidad (OMS, 2006).

La obesidad es un problema de salud que no solo se relaciona con riesgos físicos, sino que también se encuentra asociada a una gran variedad de síntomas psicológicos. Se ha señalado que las personas que padecen obesidad frecuentemente son víctimas de discriminación, lo cual impacta negativamente en su salud mental (Wadden et al., 2001) mostrando preocupaciones por el cuerpo, aislamiento social, estado de ánimo deprimido y baja autoestima (Werrij et al., 2009), una disminución en la calidad de vida 
del sujeto, aumento de prevalencia de tabaquismo, alcoholismo, depresión, ansiedad y trastornos de la conducta alimentaria, así como una mayor mortalidad (Abilés et al., 2010).

Con respecto a las consecuencias psicológicas de la obesidad severa se han reportado las siguientes: ansiedad, depresión e imagen corporal negativa, discriminación, aislamiento social y estigmatización (Wadden \& Sarwer, 2006) y que éstas a su vez pueden contribuir al desarrollo de trastornos de la conducta alimentaria, insatisfacción con la imagen corporal y depresión, siendo más vulnerables a esto las mujeres (Puhl, MossRacusin \& Schwartz, 2007).

Una de las comorbilidades psicológicas más asociadas a la obesidad es el trastorno por atracón. Se ha informado que el $24 \%$ de los pacientes con obesidad mórbida presentan trastorno por atracón severo, que a su vez se relaciona con mayor prevalencia de depresión y disminución de la calidad de vida (Alger-Mayer, Rosati, Polimeni \& Malone, 2009). Otro trastorno relacionado con la conducta alimentaria es la ingesta emocional que se presenta en el 38\% de los pacientes con obesidad y se caracteriza por comer sin hambre física, sino en respuesta a algún estado emocional (Guerdikova et al., 2007).

La obesidad grado $I V$, también llamada obesidad severa, se asocia significativamente a una mayor prevalencia de comorbilidades $\mathrm{y}$ al doble de prevalencia de mortalidad prematura que en personas con menores grados de obesidad, por lo que es de suma importancia la prevención y el tratamiento de esta condición (Martins et al., 2011).

La cirugía bariátrica ha mostrado ser la intervención más efectiva para el tratamiento de la obesidad severa con mejores resultados a largo plazo (Sjöstrom et al., 2004). Consiste en modificar quirúrgicamente ya sea la capacidad gástrica o la absorción, y los procedimientos pueden dividirse en: (a) restrictivos (limitan la capacidad gástrica, por ejemplo con banda gástrica, manga gástrica), (b) malabsortivos (se realiza una modificación anatómica para evitar que el alimento pase por alguna zona del tracto digestivo evitando que se absorba, por ejemplo, derivación biliopancreática) y (c) mixto (además de limitar la capacidad gástrica, se realiza una modificación anatómica para evitar la absorción, por ejemplo, un bypass gástrico). A pesar de los cambios anatómicos realizados por la cirugía, la pérdida de peso y el mantenimiento a largo plazo no están garantizados.

El éxito del procedimiento depende en gran medida de modificaciones conductuales por parte del paciente y de su habilidad para implementar cambios permanentes en su estilo de vida, tales como adhesión al régimen alimenticio y de actividad física, así como aprender nuevos estilos de afrontamiento para evitar acudir a la comida en momentos de tensión / estrés emocional (Bauchowitz et al., 2005).

Al respecto, se ha reportado que no todos los pacientes se benefician con la cirugía; existen diferencias significativas en las variaciones en el peso a corto y largo plazo después de la cirugía (Kinzl et al., 2006). Un ejemplo de esto es que el $20 \%$ de los pacientes que se someten a cirugía para bajar de peso no logran disminuirlo significativamente o lo recuperan en corto plazo (Greenberg, Sogg \& Perna, 2009). Este fracaso generalmente es atribuido a razones psicológicas o conductuales (Busetto et al., 2005).

Asimismo, Bauchowitz y colaboradores (2005) señalan que en las personas que no han podido implementar cambios en su estilo de vida, el resultado de la operación puede no ser óptimo e incluso puede ser contraproducente, con resultados indeseables como recuperación del peso perdido, desnutrición, depresión y ansiedad.

Se resalta la importancia del trabajo interdisciplinario, en especial del psicólogo, por lo que se ha considerado el tratamiento de la obesidad y del paciente bariátrico como un área de interés de la Psicología, ya que la obesidad severa se encuentra asociada a un aumento en la prevalencia de psicopatología. La calidad de vida de los pacientes con obesidad mórbida se ve sumamente afectada, la funcionalidad del sujeto se encuentra limitada y para que los pacientes se 
beneficien con la cirugía bariátrica y mantengan estos beneficios, es necesario un cambio en el estilo de vida del sujeto y buena adhesión terapéutica sobre las indicaciones del equipo interdisciplinario (Greenberg, Smith \& Rockart, 2004).

Se describió que la colaboración interdisciplinaria es importante para mejorar la calidad en los servicios de atención a los pacientes, además, con este enfoque se genera mayor producción de ideas, metodología y resultados de investigación (Woods \& Magyary, 2010).

El tratamiento interdisciplinario en relación a salud se refiere a valores compartidos entre las diferentes especialidades del equipo, con lo que se generan lineamientos de tratamiento y metas comunes entre los mismos (Sharp, 2006).

Actualmente las guías norteamericanas indican que el tratamiento quirúrgico de la obesidad debe ser realizado de manera interdisciplinaria, incluyendo cirujano, nutricionista, médico internista, rehabilitador físico y psicólogo (AACE / TOS / ASMBS, 2008). Si a esto se le agrega que de 1998 a 2003 el número de cirugías bariátricas realizadas anualmente se ha triplicado (de 40.000 a 146.000) mostrando una tendencia a seguir aumentando (Buchwald \& Williams, 2003), es pertinente que el psicólogo tenga conocimiento sobre el tratamiento interdisciplinario de la obesidad y el rol que juega en la cirugía bariátrica.

El objetivo del presente estudio es describir el tratamiento psicológico de la obesidad y el trabajo del psicólogo en un equipo interdisciplinario de cirugía bariátrica.

\section{EL ROL DEL PSICÓLOGO EN LA CLÍNICA DE CIRUGÍA BARIÁTRICA}

Se ha reportado que los factores psicosociales y conductuales juegan un papel fundamental en el efecto a largo plazo de la cirugía bariátrica, por lo que es de suma importancia conocerlos y modificarlos antes de la cirugía (Van Hout, Vreeswijk \& Van Heck, 2008).
El rol del psicólogo en un equipo interdisciplinario para cirugía bariátrica consiste en la evaluación inicial de los pacientes con el fin de determinar su capacidad para cumplir con los cambios en el estilo de vida que se necesitan antes y después de la cirugía, brindar al paciente psico-educación a fin de prepararlo para estos cambios y para tomar una decisión informada acerca de someterse o no a la cirugía. Esta preparación ayuda al paciente a tener expectativas realistas sobre los resultados del procedimiento al que se quiere someter y disminuir el sentimiento de incertidumbre que a su vez es generador de ansiedad. Por esa razón, la labor del psicólogo debe centrarse en la motivación y adhesión a cambios en estilos de vida a través de la aplicación de la psico-educación y técnicas cognitivo-conductuales específicas, propiciando en el paciente un sentimiento de cercanía con el equipo interdisciplinario que a su vez influye en una mejor adhesión terapéutica (Moorehead, 2008).

En relación a la evaluación inicial de los pacientes, el Instituto Nacional de Salud de Estados Unidos (NIH) en 1991 declaró que todos los pacientes que se someten a cirugía bariátrica deben someterse también a un tratamiento interdisciplinario incluyendo una evaluación a realizar por personal de salud mental (NIH, 1991), cuyo objetivo es identificar posibles obstáculos para el éxito de la cirugía. A la fecha no existen guías que definan la evaluación psicológica de estos pacientes; sin embargo, las guías norteamericanas de cirugía bariátrica (AACE / TOS / ASMBS, 2008) indican que todos los pacientes que serán sometidos a este tipo de cirugía deberán ser sometidos a una evaluación completa para identificar los factores que influyeron en el desarrollo de la obesidad, como así también aquellos factores que pudieran interferir en el bienestar del paciente después de la cirugía.

Otro de los aspectos que señalan es promover la pérdida de peso antes de la cirugía con el objetivo de disminuir el tamaño del hígado y supervisar la capacidad del paciente para seguir un régimen alimenticio. En relación a los aspectos psicológicos, se 
menciona que el paciente deberá ser sometido a una evaluación psiquiátrica y conductual encaminada al conocimiento de factores ambientales, sociales, familiares y conductuales que influyan en el problema de la obesidad y que pudieran ser obstáculo para el cambio en su estilo de vida.

El consenso mexicano de psicología bariátrica (Ríos et al., 2010), sugiere que en esta primera evaluación se debe lograr una buena empatía con el paciente y su familia y que los aspectos a ser evaluados son la presencia de psicopatología como así también su historia psiquiátrica, la historia del peso, la conciencia de enfermedad, la motivación para realizar la cirugía, el compromiso al cambio, expectativas de los resultados, redes de apoyo, conductas alimentarias incluyendo trastornos de la misma, los factores psicológicos que contribuyeron al desarrollo y mantenimiento de la obesidad y también las complicaciones psicosociales de la pérdida de peso. Sugieren también evaluar cómo ha repercutido la obesidad en los aspectos físico, emocional, social, familiar, sexual.

Por lo tanto, los dos objetivos del trabajo del psicólogo en un equipo de cirugía bariátrica son:

a.- Identificar posibles contraindicaciones para la cirugía tales como adicciones, trastornos psiquiátricos graves, trastornos psiquiátricos moderados o descontrolados. El consenso mexicano de cirugía bariátrica menciona contraindicaciones absolutas: crisis emocional actual, trastornos psiquiátricos, situaciones de riesgo (consumo de alcohol, drogas, trastorno por atracón), poco apoyo social (Ríos et al., 2010) y contraindicaciones relativas: entorno familiar disfuncional, historia previa de bulimia y/o abuso de alcohol (por lo menos 1 año en remisión), expectativas poco realistas de la cirugía y duelo reciente. No se recomienda que el paciente sea sometido a la cirugía hasta resolver esta condición si es que la presenta.

b.- Identificar retos post-quirúrgicos $\mathrm{y}$ proporcionar estrategias conductuales pa- ra la modificación del estilo de vida y el mantenimiento a largo plazo del mismo. Se debe reforzar el cambio de hábitos, apoyar el cambio en la imagen corporal y el manejo de relaciones sociales y familiares. El tiempo que sugiere el consenso mexicano de psicología bariátrica es de un año y dividido de acuerdo a las necesidades del paciente (Ríos et al., 2010).

\section{TÉCNICAS PSICOLÓGICAS MÁS UTILIZADAS PARA EL TRATAMIENTO DE LA OBESIDAD}

Existe un meta-análisis en el que se describe que el enfoque psicológico más eficaz y más utilizado en el tratamiento de la obesidad es el cognitivo conductual, y que a su vez, si se combina con tratamiento interdisciplinario muestra mejores resultados en relación a la pérdida de peso y el cambio en el estilo de vida. En lo que respecta a otro tipo de psicoterapia se ha descripto que es difícil evaluar la eficacia debido a la falta de metodología y la dificultad para replicar el tratamiento (Shaw, O'Rourke, Del Mar \& Kenardy, 2009).

A continuación se presentan brevemente las técnicas más reportadas para el tratamiento psicológico de la obesidad:

1.- Automonitoreo: Se utiliza tanto para la pérdida como para el mantenimiento del peso. Es una técnica básica para desarrollar autocontrol (Caballo, 1991) y ha sido empleada para monitorear la conducta relacionada con la meta del tratamiento (alimentación, calorías consumidas, duración del ejercicio, pasos marcados con podómetro, frecuencia cardíaca durante el ejercicio, pensamientos que sabotean la conducta del paciente, etc.) y cambiar la misma para mantener el efecto deseado. Si el paciente carece de autocontrol, la estrategia se enfoca en gratificaciones inmediatas en lugar de gratificaciones a largo plazo (como es el caso de pacientes con obesidad). Este procedimiento ha mostrado ser una estrategia eficaz para 
promover el control del peso (Wing, Tate, Reynor \& Fava, 2006) en virtud de que se ha observado que tiene efecto sobre la conducta, incrementa las conductas deseadas y disminuye las no deseadas. Además, el automonitoreo permite identificar la frecuencia o línea base de la conducta que queremos modificar, aumenta la atención del paciente hacia esa conducta y proporciona retroalimentación sobre los progresos del paciente (Butryn, Phelan, Hill \& Wing, 2007).

2.- Control de estímulos: Es otra estrategia para promover el mantenimiento del peso y consiste en modificar el ambiente a fin de incrementar el autocontrol de la conducta alimentaria (Phelan et al., 2009). Las intervenciones cognitivo-conductuales encaminadas al tratamiento de la obesidad suponen que la conducta alimentaria se rige por estímulos internos y externos que se asocian a la ingesta de cierto tipo de alimentos. La técnica de control de estímulos consiste en identificar los estímulos que desencadenan la ingesta y modificarlos. Por lo tanto, se deben modificar los estímulos que desencadenan la sobreingesta (por ejemplo, no ir a buffetes), el sedentarismo (limitar las horas 'pantalla', limitar el uso del coche) y promover el incremento de la actividad física (concurrir a un gimnasio con alguien más, utilizar medios de transporte alternos, etc.) y una alimentación más saludable, cambiando las compras semanales del supermercado (Van Dorsten, 2008). En otras palabras, el control de estímulos se dirige a la modificación del entorno del paciente.

En virtud de que el ambiente puede facilitar la aparición o no de ciertas conductas, se busca que la disponibilidad de alimentos saludables sea alta (facilitando el costo de respuesta) y que los alimentos densamente energéticos sean difíciles de conseguir para el sujeto (elevando el costo de respuesta). Parte del ambiente del paciente es su entorno social y familiar, por lo que es conveniente involucrarlos en el proceso de cambio de estilo de vida para evitar sabotaje.

3.- Solución de problemas: Este procedimiento aplicado a la obesidad consiste en enseñarle al individuo a identificar barreras para el cambio en su estilo de vida y a proponer soluciones para las mismas. Consta de cinco pasos: (a) identificar el problema (operacionalización), (b) proponer diferentes soluciones, (c) analizar los beneficios y las desventajas de cada una de esas soluciones, (d) implementar la solución que le parezca más adecuada en base al análisis anterior y (e) evaluar si esa solución fue útil o no. Se puede utilizar para involucrar redes de apoyo, para fijar horarios de alimentación, eliminar cierto tipo de alimentos, planear comidas sociales, etc.

4.- Técnicas de relajación: El significado principal y directo del término relajación es la disminución o la desaparición de la tensión. De tal modo, la noción de relajación se puede aplicar a diversas circunstancias, elementos o situaciones siempre y cuando se dé esa condición de desaparición de la tensión. Esto puede ser entendido tanto a nivel físico como corporal, emocional y psicológico. Las investigaciones han demostrado la eficacia de los procedimientos de relajación (incluyendo la relajación muscular progresiva, la meditación, la hipnosis y el entrenamiento autógeno) en el tratamiento de muchos problemas relacionados con la tensión, tales como el insomnio, la hipertensión esencial, dolores de cabeza por tensión, asma bronquial y la tensión general. Los métodos de relajación se utilizan también como tratamiento coadyuvante en muchas condiciones, por ejemplo, con la ansiedad de hablar en público, las fobias, la ansiedad intensa, el síndrome de colon irritable, el dolor crónico y la obesidad.

5.- Terapia cognitiva: Fue desarrollada por Beck en los años 60 (Beck, 2005) y postula que las interpretaciones negati- 
vas de eventos tanto internos como externos pueden llevar a una visión negativa de sí mismo, del mundo y del futuro. Los errores de interpretación o distorsiones cognitivas pueden reforzar creencias disfuncionales que tiene el sujeto e incrementar la vulnerabilidad hacia trastornos del estado de ánimo o de conducta. La premisa de la terapia cognitiva es que lo que una persona piensa sobre sí misma o cierto evento, tiene un impacto directo sobre sus emociones y su conducta. Los pacientes con obesidad tienden a presentar esquemas desadaptativos, creencias disfuncionales relacionadas con imagen corporal, ánimo deprimido, metas de peso irreales, rol desproporcionado de su peso y figura en autoevaluación así como creencias desadaptativas sobre el control de su alimentación. Si se logran modificar estos esquemas disfuncionales, se puede promover el mantenimiento de patrones de alimentación más saludables y reducir las recaídas después de perder peso (Werrij et al., 2009). Los pacientes que buscan someterse a cirugía bariátrica por lo general han probado varios métodos para intentar perder peso sin obtener resultados, por lo cual muchos de ellos desarrollan pensamientos negativos relacionados con su capacidad para seguir un régimen de alimentación, expectativas poco realistas sobre los resultados de la cirugía, etc. Por lo tanto, este tipo de pensamientos debe ser modificado para lograr una pérdida de peso y el mantenimiento del mismo.

Como se puede observar, el trabajo del psicólogo de la salud se diversifica, teniendo la oportunidad de realizar intervenciones específicas en cada momento de la problemática del paciente, donde se puede llevar un seguimiento estrecho desde la prevención, el diagnóstico de su enfermedad, seleccionando las mejores opciones de tratamiento hasta el seguimiento.

El panorama mundial actual muestra un aumento en la prevalencia de obesidad y por ende, un aumento exponencial en el nú- mero de cirugías bariátricas realizadas para su tratamiento. La norma dicta que el tratamiento debe ser interdisciplinario y en él el psicólogo debe tener un papel activo para mejorar el pronóstico de la cirugía, por lo que se resalta la importancia de aumentar el número de psicólogos preparados en el tema de la obesidad y cirugía bariátrica.

\section{REFERENCIAS BIBLIOGRÁFICAS}

Abilés, V., Rodríguez-Ruiz, S., Abilés, J., Mellado, C., García, A., Pérez de la Cruz, A. \& Fernández-Santaella, M. (2010). Psychological characteristics of morbidly obese candidates for bariatric surgery. Obesity Surgery, 20(2). 161167. http://dx.doi.org/10.1007/s11695-008-97 26-1

Alger-Mayer, S., Rosati, C., Polimeni, J.M. \& Malone, M. (2009). Preoperative binge eating status and gastric bypass surgery: a long term outcome study. Obesity Surgery, 19(2), 13945. http://dx.doi.org/10.1007/s11695-008-95 40-9

American Association of Clinical Endocrinologists / The Obesity Society / American Society for Metabolic and Bariatric Surgery (AACE / TOS / ASMBS) (2008). Medical guidelines for clinical practice for the perioperative nutritional, metabolic, and nonsurgical support of the bariatric surgery patient. Surgery for the Obesity and Related Diseases, 4, 109-184. http://dx.doi.org/10.1016/j.soard. 2008.08.009

Bauchowitz, A., Gonder-Frederick, L., Olbrisch, M., Azarbad, L., Ryee, M., Woodson, M., Miller, A. \& Schirmer, B. (2005). Psychosocial evaluation of bariatric surgery candidates: A survey of present practices. Psychosomatic Medicine, 6, 825-832. http://dx.doi.org/10.109 7/01.psy.0000174173.32271.01 
Beck, A.T. (2005). The current state of cognitive therapy: A 40-year perspective. Archives of General Psychiatry, 62, 953-959. http://dx. doi.org/10.1001/archpsyc.62.9.953

Buchwald, H. \& Williams, S.E. (2003). Bariatric Surgery worldwide 2003. Obesity Surgery, 14, 1157-1164. http://dx.doi.org/10.1381/096089 2042387057

Busetto, L., Segato, G., De Luca, M., De Marchi, F., Vianello, M., Valeri, M., Favretti, F. \& Enzi, G. (2005). Weight loss and postoperative complications in morbidly obese patients with binge eating disorder treated by laparoscopic adjustable gastric banding. Obesity Surgery, 15, 195-201. http://dx.doi.org/10.1381/09608 92053268327

Butryn, M.L., Phelan, S., Hill, J.O. \& Wing, R.R. (2007). Consistent self-monitoring of weight: A key component of successful weight loss maintenance. Obesity, 15, 3091-3096. http://dx. doi.org/:10.1038/oby.2007.368

Caballo, V. (1991). Manual de técnicas de terapia y modificación de conducta [Manual therapy techniques and behavior modification]. España: Siglo XXI.

Greenberg, I., Smith, K. \& Rockart, E. (2004). Behavioral health evaluations in bariatric surgery. Nutrition in Clinical Care, 7(1), 5-11.

Greenberg, I., Sogg, S. \& Perna, F. (2009). Behavioral and psychological care in weight loss surgery: Best practice update. Obesity, 17, 880884. http://dx.doi.org/10.1038/oby.2008.571

Guerdikova, A., West-Smith, L., McElrroy, S., Sonnanstine, T., Stanford, K. \& Keck, P. (2007). Emotional eating and emotional eating alternatives in subjects undergoing bariatric surgery. Obesity Surgery, 17, 1091-1096. http://dx.doi.org/10.1007/s11695-007-91841

Kinzl, J.F., Schrattenecker, M., Traweger, C., Mattesich, M., Fiala, M. \& Biebl, W. (2006). Psychosocial predictors of weight loss after bariatric surgery. Obesity Surgery, 16, 1609-
1614. http://dx.doi.org/10.1381/09608920677 9319301

Martins, C., Strommen, M., Stavne, O., Nossum, R., Marvik, R. \& Kulseng, B. (2011). Bariatric surgery versus lifestyle interventions for morbid obesity changes in body weight, risk factors and comorbidities at 1 year. Obesity Surgery, 21(7), 841-849. http://dx.doi.org/10.1 007/s11695-010-0131-1

Moorehead, M. (2008). Psychological evaluation. En R. Rosenthal \& D. Jones (Eds.), Bariatric surgery: A multidisciplinary approach (pp. 120-145). Edgemont, Pennsylvania: Matrix Medical Communictions.

National Institute of Health Consensus Development Conference Panel (1991). NIH conference: Gastrointestinal surgery for severe obesity. Annals of Internal Medicine, 115, 956961.

OMS (2000). Obesity: Preventing and managing the global epidemic. Report of a WHO Consultation: Geneva. Recuperado el 19 de Noviembre de 2009 de http://www.atividadefisica.pro. br/artigos/WHO\%20obesity.pdf

OMS (2006). Obesidad y sobrepeso ¿Qué son la obesidad y el sobrepeso? [Obesity and overweight. What are obesity and overweight?]. Nota Descriptiva $N^{\circ} 311$. Recuperado el 19 de Noviembre de 2009 de http://www.who.int/ mediacentre/factsheets/fs311/es/index.html

Phelan, S., Liu, T., Gordin, A., Lowe, M., Hogan, J., Fava, J. \& Wing, R. (2009). What distinguishes weight loss maintainers from the treatment seeking obese? Analysis of environmental, behavioral and psychosocial variables in diverse populations. Annals of Behavioral Medicine, 38(2), 94-104.http://dx.doi.org/10. 1007/s12160-009-9135-2

Puhl, R.M., Moss-Racusin, C.A. \& Schwartz, M.B. (2007). Internalization of weight bias: Implications for binge eating and emotional well-being. Obesity, 15(1), 19-23. http://dx. doi.org/10.103 8/oby.2007.521 
Ríos, B., Sánchez, M., Guerrero, M., Pérez, D., Gutiérrez, S., Rico, M., Balsa, M., Villalpando, J., de la Cerda, C., Silva, S., Aquino, B., Romero, V., Jiménez, L., Terán, L. \& Villalobos, E. (2010). El rol del psicólogo en la cirugía bariátrica [The psychologist's role in bariatric surgery]. Cirujano General, 32(2), 114-120.

Sharp, M. (2006) Enhancing interdisciplinary colaboration in primary health care. Canadian Journal of Dietetic Practice and Research, Fall supplement.

Shaw, K.A., O’Rourke, P., Del Mar, C. \& Kenardy, J. (2009). Psychological interventions for overweight or obesity (Review). The Cochrane Library, 1-77. http://dx.doi.org/10.1002/146 51858.CD00 3818.pub2

Sjöstrom, L., Lindroos, A.K., Peltonen, M., Torgerson, J., Bouchard, C., Carlsson, B., Dahlgren, S., Larsson, B., Narbro, K., Sjöstrom, C.D., Sullivan, M., \& Wedel, H. (2004). Lifestyle, diabetes, and cardiovascular risk factors 10 years after bariatric surgery. New England Journal of Medicine, 351(26), 2683-2693. http://dx.doi.org/10.1056/NEJMo a 035622

Van Dorsten, B. \& Lindley, E. (2008). Cognitive and behavioral approaches in the treatment of obesity. Endocrinology and metabolism clinics of North America, 37, 905-922.http://dx.doi. org/10.1016/j. ecl.2008.08.003
Van Hout, G.C., Vreeswijk, C.M. \& Van Heck, G.L. (2008). Obesity Surgery, 18(3), 321-325. http://dx.doi.org/10.1007/s11695-007-9271-3

Wadden, T.A., Sarwer, D.B., Womble, L.G., Foster, G.D., McGuckin, B.G. \& Schimmel, A. (2001). Psychological aspects of obesity and obesity surgery. Surgical Clinics of North America, 81, 1001-1024.

Wadden, T. \& Sarwer, D. (2006) Behavioral assessment of candidates for bariatric surgery: A patient oriented approach. Obesity, 14, 5362. http://dx.doi.org/10.1038/oby.2006.283

Werrij, M., Jansen, A., Mulkens, S., Elgersma, H., Ament, A. \& Hospers, H. (2009). Adding cognitive therapy to dietetic treatment is associated with less relapse in obesity. Journal of Psychosomatic Research, 67, 315-324. http:// dx. doi.org/10.1016/j.jpsychores.2008.12.011

Wing, R., Tate, D., Reynor, H. \& Fava, J. (2006) A self-regulation program for maintenance of weight loss. New England Journal of Medicine, 355, 1563-1571. http://dx.doi.org/10.1056/N EJM oa06188 3

Woods, N.F. \& Magyary, D.L. (2010). Translational research: Why nursing's interdisciplinary collaboration is essential. Research and Theory for Nursing Practice, 24(1), 9-24.

Clinica de Obesidad Hospital General Dr. Manuel Gea González México D.F. - México

Fecha de recepción: 5 de noviembre de 2012 Fecha de aceptación: 4 de septiembre de 2013 
